\title{
Laser Drop on Demand Micro Joining for High Temperature Wire Bonding Applications - System Technology And Mechanical Joint Performance
}

\author{
Stefan Stein ${ }^{1,3}$, Jasmin Dippert ${ }^{1}$, Stephan Roth $^{1,3}$ and Michael Schmidt ${ }^{1,2,3}$ \\ ${ }^{1}$ Bayerisches Laserzentrum GmbH (blz), Konrad-Zuse-Str. 2-6, 91052 Erlangen, Germany \\ E-mail: s.stein@blz.org \\ ${ }^{2}$ Institute of Photonic Technologies (LPT), Friedrich-Alexander-Universität Erlangen-Nürnberg, \\ Konrad-Zuse-Str. 3-5, 91052 Erlangen, Germany \\ ${ }^{3}$ Erlangen Graduate School in Advanced Optical Technologies (SAOT), Friedrich-Alexander- \\ Universität Erlangen-Nürnberg, Paul-Gordan-Str. 6, 91052 Erlangen, Germany
}

\begin{abstract}
Bonding wires to electrode structures e. g. of piezo actuators is a challenging task, which is currently carried out mainly by soldering or ultrasonic (US) wire bonding. Yet those bonding technologies have several drawbacks like low thermal stability of soldered bonds or high mechanical load and requirements on the surface of an electrode structure for ultrasonic wire bonding. Thus, in this paper, a drop on demand method, suitable for remote wire bonding is presented. Applying this technique, it is possible to generate droplets from $\mathrm{CuSn} 12$ preforms with a liquidus temperature of $996^{\circ} \mathrm{C}$, thus reaching significantly higher thermal stability of the joints than conventional soldering techniques. In addition, due to contact free character of the bonding technique, the mechanical loads occurring during US wire bonding can be eliminated as well. Thus the process promises significant advantages compared to the current state of the art of wire bonding. The laser drop on demand micro joining process consists of melting a braze preform by a laser pulse, a subsequent ejection of the preform from the machining head by nitrogen overpressure, followed by wetting the joining partners and cooling below solidus temperature. This results in a firm joint of electrode structure and $\mathrm{Cu}$-wire. In the scope of this paper, the shear strength of joints were measured in initial state, after thermal aging and after exposure to mechanical noise excitation, indicating shear strengths of about $22 \mathrm{MPa}$ in average, which exceeds the shear strength of standard Sn-3.5Ag respectively Sn3.5Ag-0.75Cu solder spheres with comparable diameter by about a factor of two. Thus the process shows several benefits in comparison with standard wire bonding or soldering processes particularly for high temperature applications such as high power electronics or for electronic applications in harsh environments e. g. deep drilling heads.
\end{abstract}

DOI: $10.2961 /$ jlmn.2017.03.0012

Keywords: Drop on demand, micro joining, laser droplet brazing, electronics, wire bonding

\section{Motivation}

The generation of firm bonds of $\mathrm{Cu}$-wires with thin metal layers or foils, which serve as electrode structures e.g. of solar cells or piezo actuators, is a challenge for several industries such as battery manufacturing, photovoltaic, mechatronic and electronic industry. Standard processes such as ultrasonic (US) wire bonding are limited to substrates, which withstand the mechanical stress during the bonding process, thus foils of $<20 \mu \mathrm{m}$ thickness can not be joined to wires, neither can US bonding be applied on thin metal layers on low temperature cofired ceramics (LTCCs), due to the brittle behavior of the ceramic material, particular if the metal layers exhibit thicknesses below $20 \mu \mathrm{m}$. Further, ultrasonic bonding processes require gold, silver or high purity $\mathrm{Cu}$-wires and very smooth surfaces without any imperfections, to achieve good results. In order to overcome this restrictions, a laser drop on demand bonding (LDB) process was recently developed. The particular de- 
mand for developing a laser based thermal bonding process was to enable the generation of firm bonds of solder pads of piezo actuators with Cu-conductors. The motivation for this particular approach can be derived from increasingly stringent laws regarding environmental protection which resulted in significant efforts of aeronautic and automotive industry to increase fuel efficiency of products. The most prominent approach to achieve this goal is lightweight construction. Nevertheless stiff and thin structures exhibit increased noise emission, which must be prevented in order to fulfill customers demands regarding comfort. In order to counteract noise emission, the integration of piezoelectric modules into lightweight structures is a promising noise cancelling approach for structural components [1-4].

A favorable technology to integrate piezo actuators into lightweight structures, due to its widespread application in industry, is $\mathrm{Al}$ die casting, since high surface qualities at high throughput rates can be achieved using this technology. Yet, due to the comparable high solidus and liquidus temperature (521 and $593{ }^{\circ} \mathrm{C}$, respectively) [6] of the casting alloy AlSi9Cu3(Fe), the wire bonds, connecting the piezo modules with the Cu-wires, need to withstand significant thermal loads during the die casting process. Therefore, a novel bonding process was developed, which is suitable to generate electrical interconnects of $20 \mu \mathrm{m}$ Ag-layers with $100 \mu \mathrm{m}$ Cu-wires using CuSn12 filler material with a liquidus temperature of $960{ }^{\circ} \mathrm{C}$ (value for Cu90Sn10 according to [7]) rather than standard Sn based solders. For mechanical characterization, braze droplets were deposited on solder pads consisting of screen printed Ag-layers of $20 \mu \mathrm{m}$ thickness. The solder pads are sintered onto DuPont 951 PX LTCC tape which resembles the electrode structure of a piezo actuator.

In addition, the described process provides bonds able to withstand the thermal loads occurring during the integration of piezo actuators into CFRP (carbon fiber reinforced plastics) structures $[8,9]$, whereby the consolidation of the CFRP structure is performed by an autoclave process using a maximum temperature of $230{ }^{\circ} \mathrm{C}$ for a dwell time of $2 \mathrm{~min}$, during which a consolidation pressure of $5 \mathrm{bar}$ is applied [10-12]. The laser droplet bonding process itself is based on commercially available laser droplet soldering processes shown in [13], substituting Sn based solders with Cu-base filler alloys to achieve higher thermal stability of the joints. The process hereby can be divided into four process phases. First, a spherical braze preform is inserted into a capillary and irradiated with a laser pulse which heats the braze preform above liquidus temperature. After the preform is reaching liquidus temperature, the braze droplet is expelled from the machining head via nitrogen overpressure (fig. 1).

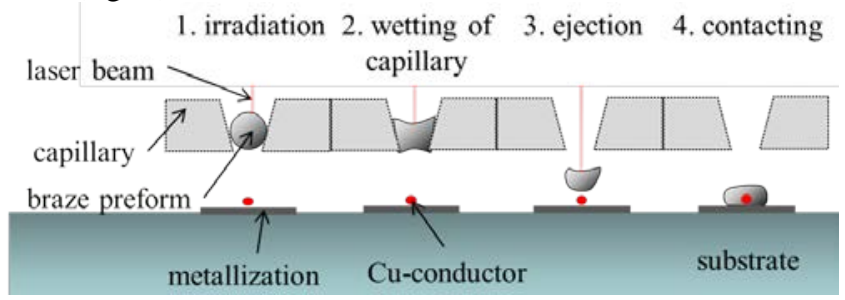

Fig. 1 Schematic of process steps [5].
Nevertheless, the required energies to melt CuSn12 braze preforms are about eight times higher than for tin based solder preforms of equal diameter. Thus, the used laser power must be scaled up, which in combination with higher heat conductivity, reflectivity and melting temperature of copper based braze results in smaller process windows as well as significantly higher heating and cooling rates of the capillary during one joining cycle. The braze alloy CuSn12 was chosen, since the alloy forms SnAg rich phases with the Ag-solderpad of the module as shown in [5] which results in a diffusion layer between Ag-pad and braze material resulting in a metallurgical bond. Fig. 2 shows $\mathrm{Cu}$ precipitations forming during cooling, resulting in an formation of AgSn rich phases at the interface between Agsolderpad structure and braze material (fig. 2).

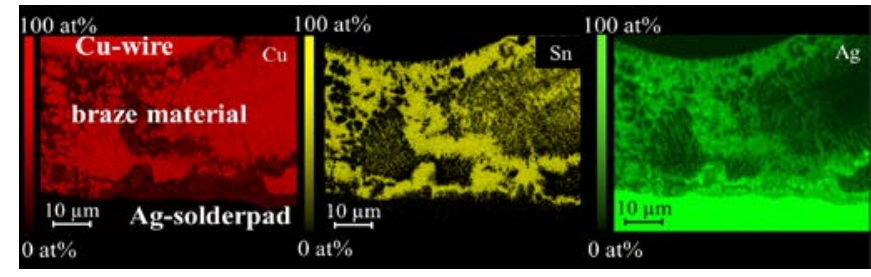

Fig. 2 Cu-segregations (left) and Ag- (right) and Sn- (middle) rich phases at the interface between Ag electrode structure and braze filler material [5].

To understand the consequences of thermal stress due to the high melting range of the filler material on the lifetime of the capillary at higher numbers of process cycles, a long term evaluation of the capillary has been carried out, to detect capillary failure or braze residues during an extended number of joining cycles.

\section{Process, system and methods}

The used CuSn12 braze preforms are generated applying an atomization process followed by subsequent sieving, which results in mass deviations of the braze preforms as stated in [8], due to the manufacturing process. This mass deviations result in varying energies necessary to be applied to melt the preforms. To analytically calculate the necessary caloric energies to melt the preforms to liquidus temperature, the physical properties of the used braze material which have been summarized in [14] are shown in table 1 .

Table 1 Physical properties of bronze (CuSn12)

\begin{tabular}{cc}
\hline Property & Value \\
\hline $\begin{array}{c}\text { Density at room } \\
\text { temperature } \sigma\end{array}$ & $\begin{array}{c}8776 \mathrm{~kg} / \mathrm{m}^{3} \text { (by the } \\
\text { rule of mixtures based } \\
\text { on [15]) }\end{array}$ \\
$\begin{array}{c}\text { Specific heat at room } \\
\text { temperature } C_{p}\end{array}$ & $\begin{array}{c}367 \mathrm{~J} /(\mathrm{kg} \mathrm{K}) \text { (by the rule } \\
\text { ofixtures based on } \\
\text { [15]) }\end{array}$ \\
$\begin{array}{c}\text { Enthalpy of fusion } \\
\Delta H_{f}\end{array}$ & $91,3 \mathrm{~kJ} / \mathrm{kg}[16]$ \\
Liquidus temperature, & $1269 \mathrm{~K}[15]$ \\
$T_{L}$ & \\
\hline
\end{tabular}


The necessary caloric energy $E_{0}$ to liquefy the braze volume is consisting of the energy, which is necessary heat the braze preform from room to liquidus temperature, to which the energy necessary for phase transition needs to be added (eq. 1):

$$
E_{0}=c_{p} m \Delta T+\Delta H_{f} \cdot m
$$

In eq. $1 c_{p}$ indicates the specific heat at room temperature, $\Delta T$ the temperature difference between room- and liquidus temperature of the braze alloy, $m$ the mass of the used braze preform and $\Delta H_{f}$ the enthalpy of fusion of the braze alloy. In order to evaluate the influences of mass deviations of the preforms on the necessary energies, a random sample of 100 preforms were balanced. The results of the measurements and calculations are shown in table 2.

\begin{tabular}{cc}
$\begin{array}{l}\text { Table } 2 \text { Diameter, mass and necessary energies of used } \\
\text { braze preforms }(\mathbf{n}=\mathbf{1 0 0})\end{array}$ & Value \\
\hline Property & $638.8 \pm 19 \mu \mathrm{m}$ \\
\hline Diameter & $1.201 \mathrm{mg} \pm 0.108 \mathrm{mg}$ \\
Average Mass & $1.34 / 0.65 \mathrm{mg}$ \\
Maxim/Minimum Mass & $0.41 \pm 0.03 \mathrm{~J}$ \\
Mean Energy & $0.48 / 0.23 \mathrm{~J}$ \\
Maximum/Minimum & \\
\hline
\end{tabular}

The standard deviations of the necessary energies are relatively low, nevertheless the spread between lowest and highest masses respectively energies vary significantly, thus, the pulse length needs to be adjusted to the braze preform condition, which is carried out by applying an online process control able to adjust the pulse length according to the precondition of the braze preform as explained in [5]. The implemented process control is capable to interrupt the laser pulse within $<2 \mu \mathrm{s}$, which is sufficiently fast to avoid substrate perforations. After thorough melting of the preform, the braze droplet is ejected from the capillary by nitrogen overpressure. After a flight phase the droplet impinges on the joining area consisting of the electrode of the piezo module and the $\mathrm{Cu}$-wire, wetting both and forming a firm joint (see fig. 1 and 2).

\subsection{Experimental setup and beam source}

The laser used for the experiments is a fiber laser with a wavelength of $1070 \mathrm{~nm}$, a maximum optical output power of $200 \mathrm{~W}$ and a raw beam diameter of $6.9 \mathrm{~mm}$, which exhibits four times higher optical output power than standard solder ball bumping systems commercially available. It is focused with a lens of focal length $f=50 \mathrm{~mm}$ to form a laser spot of $2 \mathrm{w}_{0}=10.9 \mu \mathrm{m}$. The setup is operated at focus offset to obtain a beam diameter of $600 \mu \mathrm{m}$, which correlates with the preform diameter. This measure is necessary to avoid a perforation of the braze preform by intense laser radiation, which would result in the formation of a keyhole known from deep penetration welding, which leads to un- balanced and fragmentary braze ejection and thus clogging of the capillary.

The used capillary consist of zirconia toughened aluminum oxide ceramic (ZTA) with a lower thermal conductivity than most other technical ceramics of about $24 \mathrm{~W} / \mathrm{mK}$, which proved suitable in comparison to other technical ceramics like aluminum nitride $\left(\mathrm{AlN}-\lambda_{\text {Therm }}=180 \mathrm{~W} / \mathrm{mK}\right)$, or silicon nitride $\left(\mathrm{Si}_{3} \mathrm{~N}_{4}-\lambda_{\text {Therm }}=90 \mathrm{~W} / \mathrm{mK}\right)$. In preliminary investigations it was shown that high thermal conductivities of AlN or $\mathrm{Si}_{3} \mathrm{~N}_{4}$ results in significant heat loss of the braze melt due to heat conduction, which inhibits the droplet from detachment. Thus ZTA was chosen as capillary material due to its comparably low thermal conductivity.

A coaxially mounted camera enables to observe capillary and joint position and enables a precise alignment of the machining head to the desired joint position. Further, the image can be used to monitor the capillary condition regarding wear or the development of braze residues. To enable capillary monitoring, an image processing algorithm was developed, which uses camera images acquired coaxially.

First an image of the capillary is acquired, using the focusing optic as imaging element to focus the capillary plane on the camera sensor. The necessary z-translation of the focusing optic is carried out, using a step motor, in order to achieve a high degree of automatization without the need of manual manipulation of the optical system. The minimum resolution of the z-translation stage is determined by the repetitive accuracy of the used limit switch of $10 \mu \mathrm{m}$, which is used for referencing the position of the optics. Thus the resolution of the z-translation is $8.3 \%$ of the Rayleigh length of the focused laser beam of $83 \mu \mathrm{m}$, which provides a sufficiently high resolution to adjust the focus diameter to the braze preform diameter.

This approach significantly increased repeatability of the alignment between laser spot and capillary orifice in x-, $\mathrm{y}$ - and z-direction between experiments, avoiding capillary perforations originating due to manual alignment deviations. A quantification, how this measure can enhance capillary standing times was carried out in the scope of this paper. A nitrogen feed attached to a pressure regulator enables the application of a defined nitrogen overpressure inside the machining head against ambient pressure. The overpressure is monitored in situ by a pressure sensor.

\subsection{Mechanical characterization}

For mechanical characterization, braze droplets were deposited on solder pads consisting of printed Ag-layers of $20 \mu \mathrm{m}$ thickness. The solder pads are sintered onto DuPont 951 PX low temperature cofired ceramic (LTCC) substrates Joints were deposited onto the electrode structure and subsequently sheared from the LTCC substrate, using a XYZTEC shear tester Condor 150-3. The velocity of the shear chisel was $5 \mathrm{~mm} / \mathrm{s}$. The measurement precision is $0.1 \%$ of the absolute value. Subsequently, the area at which failure occurred was measured using a laser scanning microscope OLYMPUS OLS 4000 and the maximum 
force at failure $F_{\text {Max }}$ was divided for each joint by the area of fracture $A_{\text {Failure }}$, to calculate the shear strength $\tau_{\text {Shear }}$ according to eq. 2.

$$
\tau_{\text {Shear }}=\frac{F_{\text {Max }}}{A_{\text {Failure }}}
$$

In order to quantify the joint strength, first the initial shear strength of the joints were measured, to obtain a reference. Since piezo modules are applied e.g. to achieve active vibration damping of lightweight structures, the electrical bonds must endure mechanical loads induced by vibration of the structure which they are integrated in. In order to evaluate the influence of mechanic vibration on the shear strength, joints were exposed to vibrations using an electrodynamic vibration exciter RMS SW 1512 according to industrial standard DIN EN 60068-3-8. The samples are exposed to effective accelerations $a_{\text {effektive }}=3.88 \mathrm{~m} / \mathrm{s}^{2}$ applying a noise signal in $\mathrm{x}-$, $\mathrm{y}$ - and $\mathrm{z}$ direction for 8 hours. Particularly in automotive or aircraft body applications structures are exposed to frequent temperature changes during lifetime, thus it is important to evaluate the impact of cyclic thermal loads on the mechanical properties of the generated joints. In order to evaluate the effect of cyclic thermal loads on the mechanical strength, the joints were exposed to thermal cycles carried out according DIN EN 60068-2-14 (1000 cycles $40^{\circ} \mathrm{C} /+125^{\circ} \mathrm{C}$ dwell time: $10 \mathrm{~min}$, transfer time: $10 \mathrm{~s}$ ).

A quantification of the shear strength of the bond necessary to resist the forces arising during the casting process is not possible, since the forces depend on the flow rate and viscosity of the $\mathrm{Al}$ melt and thus represent a fluid dynamics problem, which is dependent on several factors e.g. the geometry of the casting mold. In addition, the bond is encapsulated with epoxy resin to prevent shortcuts of the electrode structures before casting. Thus only thermal requirements can be formulated on the bond itself. Nevertheless, a quantification of mechanical load bearing capabilities of such bonds must be carried out, since the described bonding method can also be used for other joining problems where high bonding strengths are required.

\section{Results and discussion}

\subsection{Long term capillary behavior}

Since the capillary is the part of the setup, which is exposed to significant heating and cooling rates of about $22100 \mathrm{~K} / \mathrm{s}$ [17] during a single joining cycle, several mechanisms, such as wear, the formation of cracks or clogging of the capillary with braze residues are to be expected during large numbers of process cycles. In order to be able to take countermeasures if such effects occur, the capillary needs to be monitored to ensure reproducible joint results, which are crucial for bonding processes in large scale electronics packaging. In order to monitor the capillary, an image processing algorithm is applied, utilizing the coaxially aligned camera. For image acquisition, and to assure maximum image contrast, the focal plane of the focusing lens must match with the upper surface of the capillary to image the capillary plane on the camera. Subsequently, the image is acquired and binarized, by applying a conversion threshold. Fig. 3 shows a coaxially acquired image of an unused capillary and the binarized image after thresholding, showing the orifice of the capillary (A) and the surrounding of the machining head $(\mathrm{B})$.

The threshold value must be determined by evaluating the complete set of recorded images during the experiment. This must, e. g. due to changing lighting conditions, be carried out for all sets of experiments individually. For the described case, the threshold value was chosen to be 0.7 , meaning only pixels with values above $70 \%$ of the total value range of an 8 bit grayscale image of 0 (black) to 255 (white) are considered illuminated and thus represent the capillary orifice.
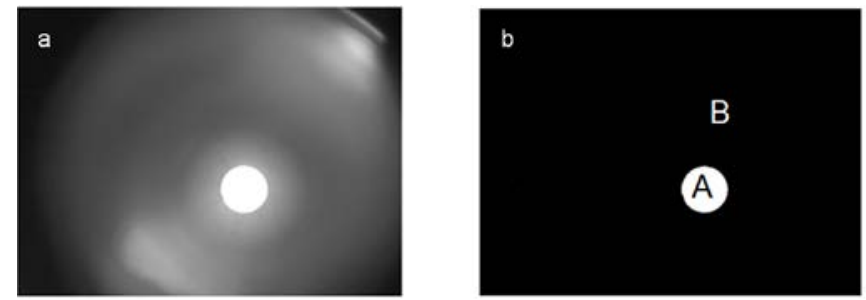

Fig. 3 Picture of capillary orifice in initial state (a) and after binarization (b) with two discrete areas: capillary (B) and capillary aperture (A).

Next the convex hull encircling the capillary aperture of the binarized image is calculated, omitting areas smaller than 7 neighboring white pixels in order to avoid hot pixels influencing the evaluation. The resulting binary image will now consist of one area, which is representing the orifice of the capillary (A) in fig. 3. Subsequently, the smallest circle enclosing the convex hull of the capillary aperture is calculated. In order to do this, a circle is gradually decreased in size, until three points of its circumference correspond with white pixels of the binarized capillary image. The number of three pixels was selected, since three coordinates are the minimum number to describe a circle exactly.

The area of this smallest inbound circle is subsequently compared with the initial binarized image of the capillary, which allows a statement regarding the deviation in area percent of the actual image of the capillary in comparison with the perfect circle. The deviation in area percent, respectively number of pixels enables a quantification of the development of braze residues inside the capillary orifice. Thus a percentage of e.g. $30 \%$ must be interpreted in a way that $70 \%$ of the capillary orifice are free of contaminations with braze residues respectively $30 \%$ of the capillary is clogged with braze residues. Fig. 4 shows exemplary images for capillary at different conditions and the response of the algorithm. 




Fig. 4 Image (top) and response (bottom) of the algorithm for unused capillary (a) and exemplary images of capillaries with braze residues (b, c).

The image processing algorithm was calibrated using a perfect circle. For unused capillaries (image a in fig. 4) the algorithm calculates an area fraction to be blocked of about $0.1 \%$, depending on the lighting conditions, which thus represents the resolution limit of the algorithm. With the image processing algorithm it is possible to evaluate the formation of braze residues inside the capillary over a large number of joining cycles. Fig. 5 shows the evolution of braze residues inside the capillary within 2,000 joints.

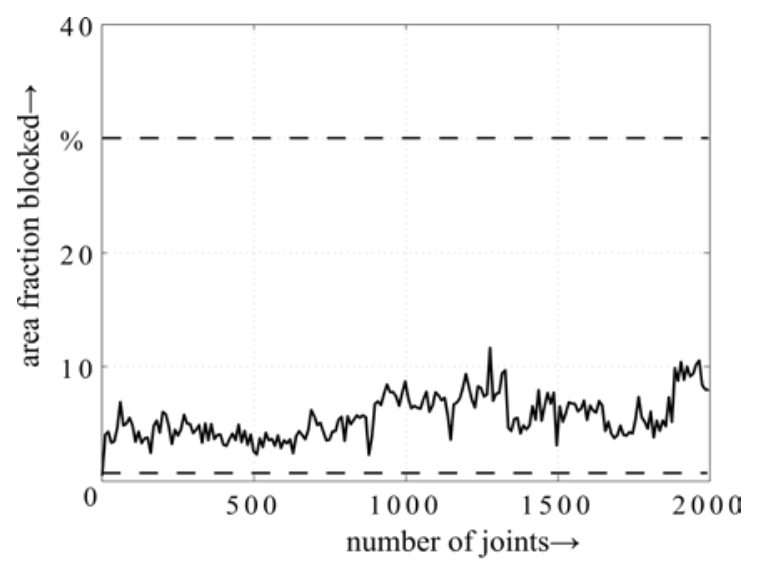

Fig. 5 Evolution of braze residues within 2000 process cycles ( pas $_{\text {Gas }} 120 \mathrm{mBar}$; $\left.\mathrm{P}_{\text {Laser }}=140 \mathrm{~W}\right)$.

The upper dashed line at $30 \%$ was found in preceding investigations [5] to be a threshold value, demanding manual clearing or a replacement of the capillary. The lower dashed line indicates the resolution limit of the imaging system. In contrast to former results [5], capillary failure or clogging could not be observed within 2,000 joining cycles, which can be accounted to the poor wetting behavior of the ZTA capillary for CuSn-braze material, which enforces a throughout ejection of the braze droplet from the capillary. In addition, carrying out the z-translation of the focusing optic, using a step motor significantly increased the repeatability lateral position of the laser spot in respect to the capillary orifice, which helps to avoid capillary damage due to misalignment of the optical system. The development of capillary residues is not exceeding $15 \%$, which was shown to be sufficient for reproducible joint results in [5]. Partial clogging occurs, nevertheless it is not a permanent effect, since subsequent joining cycles clear the capillary orifice from braze residues indicated by fig. 5 . During the experiment, no capillary failure was detected, neither manual cleaning of the capillary was necessary.

\subsection{Mechanical strength}

\subsubsection{The influence of pulse power and capillary over- pressure on the shear strength}

In order to understand the influence of mechanical vibration or cyclic thermal loads on the joint strength, first the initial shear strength of the joints were quantified, to obtain a reference. The results are shown in fig. 6 and 7 in which the shear strength is plotted over the applied pulse power respectively the nitrogen overpressure applied to the machining head. This approach enables to evaluate the influence of both parameters on the shear force independently.

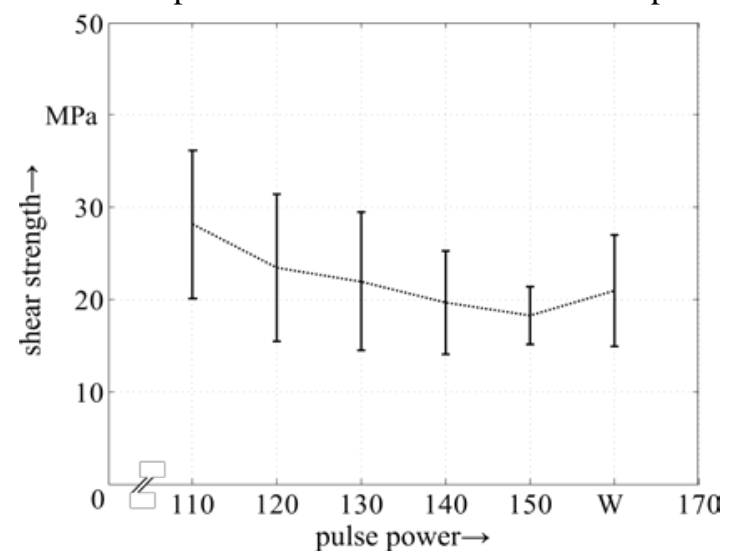

Fig. 6 Shear strength over pulse power in initial state (substrate: DuPont 951 PX LTCC tape , $20 \mu \mathrm{m} \mathrm{Ag-}$ metallisation, $\mathrm{N}=7$ ).

By increasing the pulse power from $110 \mathrm{~W}$ to $160 \mathrm{~W}$, the average shear strength is reduced from $28.1 \mathrm{MPa}$ to 20.9 MPa, resembling a reduction of shear strength of $25.6 \%$ which is most likely to be caused by an overheating of the braze preform resulting in higher residual stress in the joining area after cooling and thus shrinkage of the braze material. The quantification of the residual stress due to thermal shrinkage can be determined by electron backscatter diffraction (EBSD) analysis of the crystal lattices in at the interface between Ag-electrode and braze material, which is topic of future works.

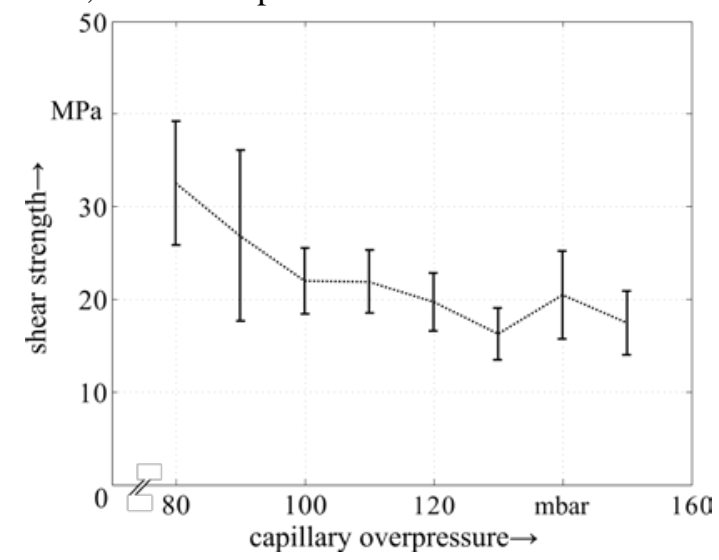

Fig. 7 Shear strength over capillary overpressure in initial state (substrate: DuPont 951 PX LTCC tape, $20 \mu \mathrm{m}$ Agmetallisation, $\mathrm{N}=7$ ). 
The average shear strength was found to be $22.1 \mathrm{MPa}$, which exceeds the shear strength of standard $\mathrm{Sn}-3.5 \mathrm{Ag}$ respectively $\mathrm{Sn}-3.5 \mathrm{Ag}-0.75 \mathrm{Cu}$ solder balls with comparable diameter by about a factor of 2 [19]. By increasing the overpressure from 80 mbar to 150 mbar the average shear strength is reduced from $32.8 \mathrm{MPa}$ to $17.2 \mathrm{MPa}$, respectively a reduction of shear strength of $47.6 \%$. Thus the influence of the overpressure on the resulting shear strength is evident and is accounted to the fact, that the gas impulse on the solidifying droplet alters its geometry resulting in a larger bonded area, which is critical due to the shrinkage of the CuSn12 braze which induces stress into the brittle ceramic. A statistical significance of the observed mechanism is however critical to be deducted from the measurements, due to the comparably high standard deviation, which must be attributed to the brittle behavior of the LTCC substrate.

Using a capillary overpressure of 100 mbar seems to be the best compromise regarding high shear strength and yet comparably low standard deviations. The mean standard deviation of $5.5 \mathrm{MPa}$, representing $24.9 \%$ of the absolute value can be considered significant. Regardless, the shear strength of the obtained joints is by about a factor of 2 higher than the shear strengths generated with Sn based solder preforms as investigated by [19]. Failures mainly occurred in the ceramic substrate, leaving shell shaped craters. Fig. 8 shows a three-dimensional LSM measurement of the topography of the ceramic after the shear force at failure was determined. Due to the mechanical shear force applied during testing, the braze material and Agmetallisation is being ripped out of the ceramic substrate, leaving a characteristic shell shaped crater with a depth of $170 \mu \mathrm{m}$ in the LTCC substrate (fig. 8).

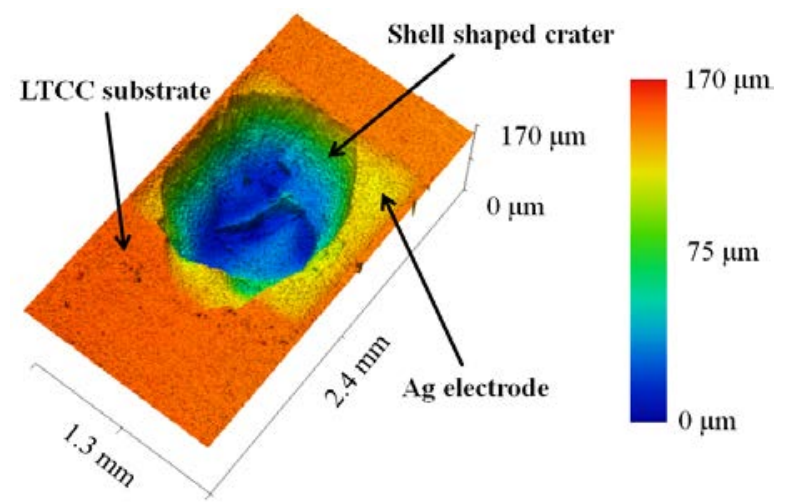

Fig. 8 Topography of crater in the LTCC substrate after shear testing; Ag electrode was partially removed during shear testing ( (Gas $_{\text {Ga }} 120 \mathrm{mBar}$; $\left.\mathrm{P}_{\text {Laser }}=140 \mathrm{~W}\right)$.

The failures of the LTCC matrix can be explained by its inherent brittleness, which makes ceramics the most critical material for obtaining reliable joints [20, 21]. Since the measured area is considered when calculating the shear strength (eq. 2) the arbitrary shape of the craters in the ceramic substrate due to chipping increases the standard deviation of the measured shear strengths, which is reflected by the results in section 3 by the large standard deviations of the measured values.

Since the difference between ceramic and metal thermal expansion coefficients (CTE) leads to the development of residual thermal stresses during cooling of the braze material to room temperature, this effect is to be held responsible for a reduction of the joint strength [21]. Thus the thermal stress during cooling and shrinkage of the filler material in combination with the high elastic module and low-relaxation characteristics of the ceramic substrate, preventing a relief respectively redistribution of the stresses, which results in residual stresses in the joining area [21] particularly between Ag-electrode and LTCC due to the ductile behavior of the Ag-pad, which can not rule out the shrinkage of the braze filler material. Therefore, the Ag-pad translates said residual stress into the LTCC matrix, which results in microscopic shell shaped cracks of the ceramic particularly at the outer circumference of the bonded interface (highlighted by the dashed line in fig. 9).

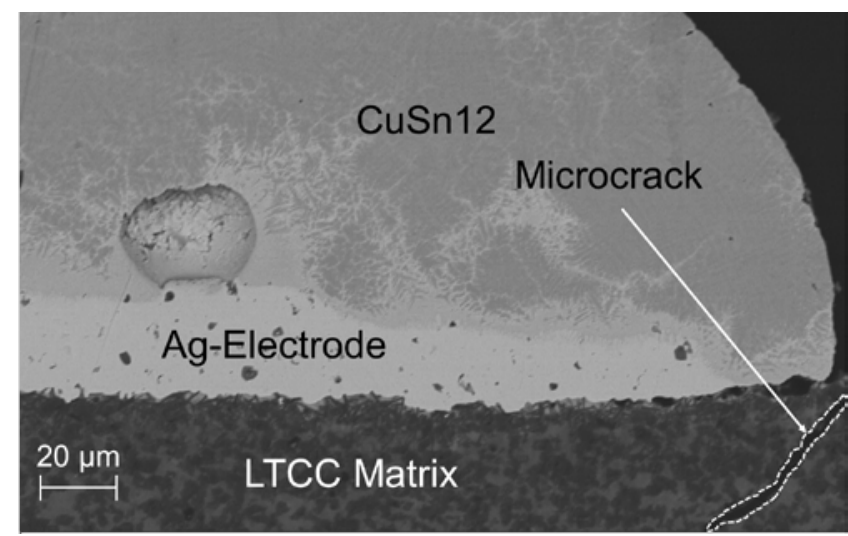

Fig. 9 SEM image of joint cross section with microcrack (substrate: DuPont 951 PX LTCC tape, $20 \mu \mathrm{m}$ screen printed Ag-metallisation, EHT=20 kV; Mag. 1.55K x).

Such micro cracks act as failure initiators when exposed to mechanical loads [22] which as a result determines the maximum mechanical load a bond is able to bear. Due to the discrepancy of material properties, particularly the interface of brittle and ductile layers between Agelectrode and LTCC is prone to mechanical failure [19, 23] and therefore can explain the observed high standard deviations. The pulse power seems not to have a significant influence on the mechanical strength of the joint, yet the overpressure has a larger impact on the mechanical strength, since increasing overpressure results in a decreased droplet height, resulting in larger tethered interface of droplet and Ag-electrode structure. This effect increases the induced mechanical stress during cooling due to a mismatch of the coefficient of thermal expansion [21] between LTCC substrate and braze droplet and can explain the reduction of the shear strength for increasing gas overpressures. The bonded area $A_{\text {Bond }}$ can be described as by a linear relationship as a function of gas overpressure by equation 3 , which has been determined using a curve-fitting algorithm, applying the sum of least squares method on the mean droplet area measured:

$$
A_{\text {Bond }}\left[\mathrm{mm}^{2}\right]=0.006852 \frac{\mathrm{mm}^{2}}{\mathrm{mbar}} * P_{\text {Nitrogen }}-0.3052 \mathrm{~mm}^{2}
$$

According to eq. 3 with increasing overpressure, the joining area gradually increases. However it must be stated, that this equation is only valid in the range between 80 and 
180 mbar and must be taken as an approximation, due to the large standard deviations of the measured bonding areas. However, the joint weakening due to initiation of micro cracks by shrinking filler material seems to surpass a gain of mechanical loadbearing by increasing the bonded interface.

\subsubsection{The influence of pulse power and capillary over- pressure on the shear strength after tempera- ture cycle tests}

As motivated and explained in 2.3 the joints were exposed to thermal cycles according DIN EN 60068-2-14 (1000 cycles $-40^{\circ} \mathrm{C} /+125{ }^{\circ} \mathrm{C}$ dwell time: $10 \mathrm{~min}$, transfer time: $10 \mathrm{~s}$ ). The exposed joints were generated with similar parameters as described in section 3.2.1 further, mechanical characterization was carried out as explained in section 2.2 by measuring the maximum force at fracture, which is divided by the area at which failure occurs (see fig. 8). The area of fracture and the maximum force at fracture are in the process measured for each joint individually. The results are shown in fig. 10 and 11 .

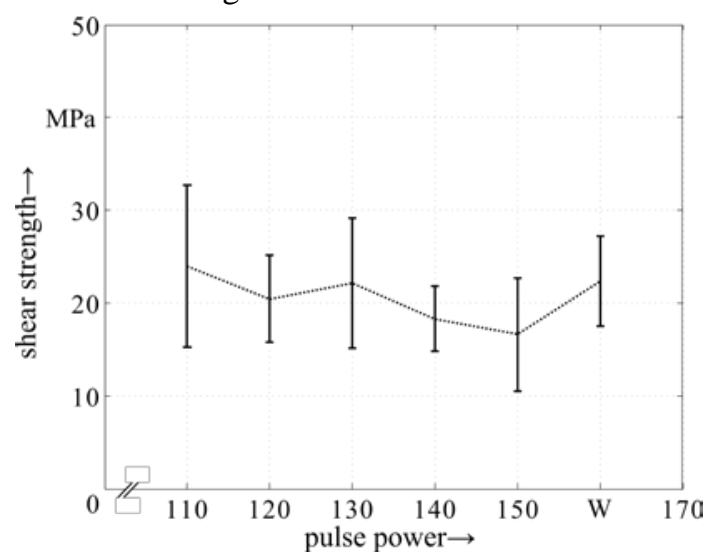

Fig. 10 Shear strength over pulse power after thermal cycle test according to industrial standard DIN EN 60068-2-14 (1000 cycles $-40^{\circ} \mathrm{C} /+125^{\circ} \mathrm{C}$; dwell time: $10 \mathrm{~min}$, transfer time: $10 \mathrm{~s}$; substrate: DuPont 951 PX LTCC tape, $20 \mu \mathrm{m}$ Ag-metallisation, $\mathrm{N}=7$ ).

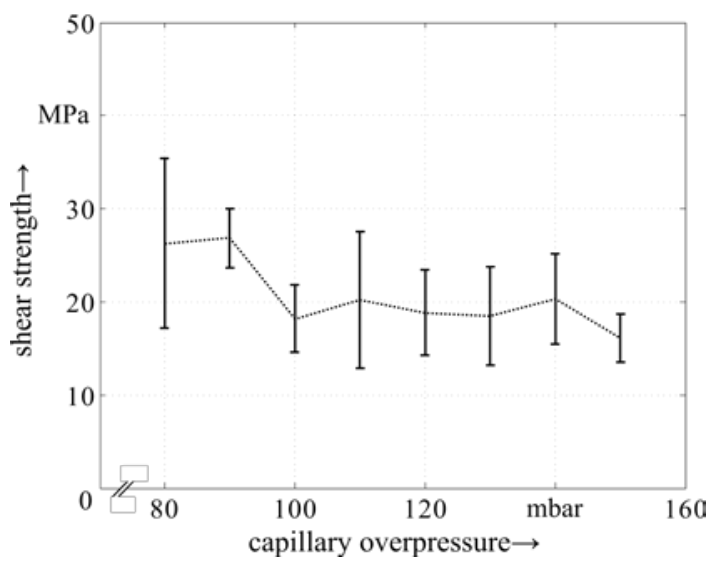

Fig. 11 Shear strength over capillary overpressure after thermal cycle test according to industrial standard DIN EN 60068-2-14 (1000 cycles $-40^{\circ} \mathrm{C} /+125^{\circ} \mathrm{C}$; dwell time: $10 \mathrm{~min}$, transfer time: $10 \mathrm{~s}$; substrate: DuPont 951 PX LTCC tape, 20 um Ag-metallisation, N=7).
The results seem to confirm the findings of the reference group, indicating high standard deviations, which must be accounted to the fact that failure occurs in almost all cases in the ceramic, which is obviously the weakest point of the joint. By increasing the pulse power from $110 \mathrm{~W}$ to $160 \mathrm{~W}$, the average shear strength is reduced from 24.0 $\mathrm{MPa}$ to 22.3 $\mathrm{MPa}$, resembling a reduction of shear strength of $7.0 \%$.

Thus, the influence of pulse power seems neglectable, since it does not have a significant influence on the droplet geometry. Contrarily, by increasing the overpressure from 80 mbar to 150 mbar, the average shear strength is reduced from 26.2 $\mathrm{MPa}$ to $16.1 \mathrm{MPa}$, resembling a reduction of shear strength of $38.5 \%$, which indicates a loss of mechanical strength; however, the large standard deviation must be taken into account.

This is triggered by the same mechanism as explained above, since a larger tethered interface between braze droplet and Ag-pad results in increased residual stresses in the ceramic substrate during cooling and shrinkage of the braze material. The mean value of the shear strengths after applying cyclic thermal loads was 20.6 MPa with a standard deviation of $5.4 \mathrm{MPa}$ representing $26.2 \%$ of the absolute value. In comparison with the mean shear strength without exposure to thermal loads of $22.1 \mathrm{MPa}$ this represents a reduction of $6.8 \%$, which represents a surprisingly low reduction. Again a statistical significance of the observed mechanism should not be deducted from the measurements, due to the comparably high standard deviation, which must be attributed to the brittle behavior of the LTCC substrate, which results in arbitrary shapes of the sheared off areas (see fig. 8). In fact, in most of the cases, the complete Agpad was sheared off the ceramic substrate, which results in an exceedingly large area of failure, which results in a lower shear strength calculated according to eq. 2.

However it must be stated, that thermal cycles seem not to reduce the joints mechanical strength significantly, which poses considerable advantages over soldered bonds in respects of shear strengths and temperature stability, thus the use of copper-based filler material is to be favored over Sn-based solders in high temperature conditions.

\subsubsection{The influence of pulse power and capillary over- pressure on the shear strength after mechanical vibration tests}

In order to evaluate the influence of mechanic vibration on the shear strength, joints were exposed to vibrations using an electrodynamic vibration exciter as explained in 2.3. The joints were generated with similar parameters as in section 3.2.1. and 3.2.2. Mechanical characterization was carried out as described in previous sections. The results are shown in fig. 12 and 13. 


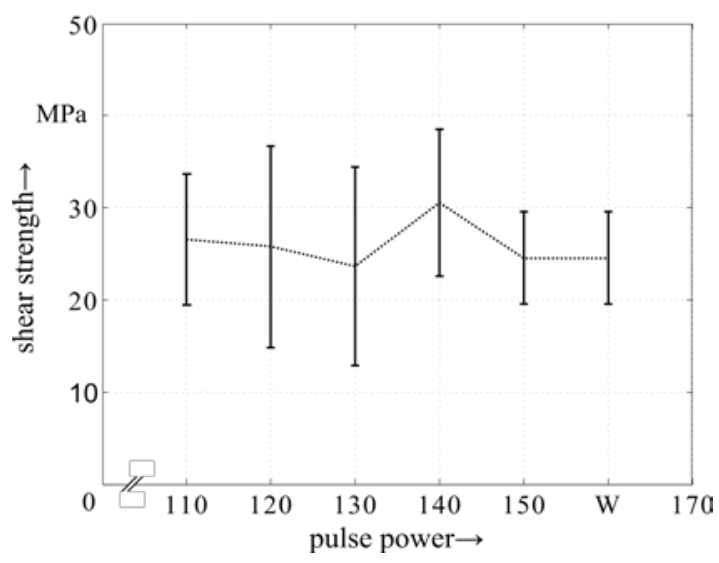

Fig. 12 Shear strength over pulse power after exposition to acceleration according to DIN EN 60068-3-8

( $a_{\text {effektive noise: }} 3.88 \mathrm{~m} / \mathrm{s}^{2}$; excitation in $\mathrm{x}-, \mathrm{y}-$ and $\mathrm{z}-$ direction: 8 hours; substrate: DuPont 951 PX LTCC tape; $20 \mu \mathrm{m}$ Ag-metallisation; $\mathrm{N}=7$ ).

Apparently, increasing the pulse power from $110 \mathrm{~W}$ to $160 \mathrm{~W}$ does not have a significant statistical influence on the resulting shear strength, which is reduced from 26.6 $\mathrm{MPa}$ to $24.5 \mathrm{MPa}$, resembling a reduction of shear strength of $7.9 \%$. Thus, the influence of pulse power on the resulting shear strength can be considered low, particularly when taking the large standard deviation of the measured values into consideration. The situation changes when increasing the overpressure from 80 mbar to 150 mbar.



Fig. 13 Shear strength over capillary overpressure after exposition to acceleration according to DIN EN 60068-3-8 ( $a_{\text {effektive noise }}: 3.88 \mathrm{~m} / \mathrm{s}^{2}$; excitation in $\mathrm{x}-$, $\mathrm{y}-$ and $\mathrm{z}$ direction: 8 hours; substrate: DuPont 951 PX LTCC tape; $20 \mu \mathrm{m}$ Ag-metallisation; $\mathrm{N}=7$ ).

The average shear strength is reduced significantly from 36.7 $\mathrm{MPa}$ to 23.2 $\mathrm{MPa}$, resembling a reduction of shear strength of $38.5 \%$. Thus, the influence of the overpressure on the resulting shear strength again seems to have a larger impact than the pulse power, which can be attributed to increased bonding area between electrode structure and braze material which favors the formation of residual stress, which induce said micro cracks at the edges of the bonded areas in the ceramic substrate. Since in the majority of the cases the strength of the joint exceeded the strength of the ceramic substrate, failure occurs in the ceramic, since the joint with the metallisation pad was ripped out of the ceramic, leaving a shell shaped crater with shear cracks. The arbitrary shape of that brittle failure in the ceramic significantly influences the results of the measurements, since larger failure area decreases the shear strength of the joint (see fig. 8). The pulse power seems not to play a significant role since its influence on the joint geometry is not dominant in contrast to the capillary overpressure, which significantly alters the droplet geometry and thus the bonded area due to a flattened droplet shape. As a result, the capillary overpressure seems to have a more dominant effect on the shear strength. The underlying effect describing this behavior is believed to be the same as explained in section 3.2.1. The mean value of the shear strengths after mechanical vibration test was $25.9 \mathrm{MPa}$ with a standard deviation of 7.3 MPa, resembling $28.2 \%$ of the absolute value without exposure to vibrations. In comparison with the initial shear strength, the shear strength was increased by $17.2 \%$. The increasing shear strength must be accounted to statistical effects occurring due to the large standard deviations, since the mean values of both series of measurements are well within the confidence interval. In summary, a reducing effect on the bonds shear strength after exposure to vibrations cannot be concluded from the measurements.

\section{Summary and outlook}

Laser based drop on demand micro joining is a novel flux less approach for thermally stable joining of thin metal layers or foils with wires or conductors. In the scope of the experiments the endurance of the used capillary exceeded 2000 joining cycles without showing fatigue or material failure, neither was clogging of the capillary with braze material observed. During the experiment, neither manual cleaning of the capillary, nor its replacement was necessary. In addition, the shear strengths of the generated joints have been measured after thermal cycle test according to industrial standard DIN EN 60068-2-14, after vibration testing according to industrial standard DIN EN 60068-3-8 as well as in initial state. The measured average shear force at failure for all tested specimen was 22.1 $\mathrm{MPa} \pm 5.5 \mathrm{MPa}$, which exceeds the force at fracture of standard $\mathrm{Sn}-3.5 \mathrm{Ag}$ respectively $\mathrm{Sn}-3.5 \mathrm{Ag}-0.75 \mathrm{Cu}$ solder balls with comparable diameter by about a factor of 2 [19]. A relatively low influence of the pulse power on the maximum shear strength was found, yet the overpressure inside the machining is playing a more dominant role regarding the mechanical strength of the joint. The underlying effect is a larger wetted and thus bonded area when working at higher overpressures due to a flatter droplet shape. A larger bonding area results in the emergence of mechanical stress during cooling and shrinkage of the braze material due to CTEmismatch between LTCC and braze material. This residual stress building up can result in the formation of microcracks at the brittle LTCC, particularly at the edges of Ag-electrode and ceramic (see fig. 9) which thus represent predetermined points of failure. This effect determines the maximum load-bearing capabilities of the joint.

It must be stated, that the measured values of the shear strengths indicate rather the mechanical strength of the ceramic substrate, than the shear strength of the Agelectrode structure with the braze material, since failure occurs mainly in the ceramic substrate, which shows a brit- 
tle failure behavior. In the majority of the cases, the complete Ag-pad was ripped out of the ceramic substrate, which indicates that the calculated shear strengths underestimate the real shear strengths of the bond (see eq. 2). However, lower pressures enable higher mechanical strengths of the generated joints, since the bonded area is smaller and thus the residual stress induced into the ceramic substrate during shrinkage of the braze material is less pronounced.

In addition, the measurements indicate a negligible influence of vibration excitation and cyclic thermal loads on the mechanical integrity of the joints, thus laser drop on demand micro joining is a promising contactless bonding approach for harsh environments or high power applications and can be applied on electrode structures or foils with thicknesses of $20 \mu \mathrm{m}$ and below. In future works microscopic qualitative analysis of the occurring diffusion processes between CuSn12 filler material and Ag solder pads will be carried out by applying electron dispersive Xray spectroscopy, in order do analytically describe the influence of the process parameters on the occurring interdiffusion which determines the bond quality between Agelectrode structure and braze material. The suitability of the drop on demand joining method was shown for piezo actuators being integrated into Al-structures by $\mathrm{Al}$ die-casting in [8] and proofs the high thermal stability of the generated joints.

\section{Acknowledgments and Appendixes}

This research is supported by the Deutsche Forschungsgemeinschaft (DFG) in context of the Collaborative Research Centre/Transregio 39 “Großserienfähige Produktionstechnologien für leichtmetall- und faserverbundbasierte Komponenten mit integrierten Piezosensoren und aktoren“ PT-PIESA, subproject A04. In addition, the authors gratefully acknowledge cooperation with the Erlangen Graduate School in Advanced Optical Technologies (SAOT) by the Deutsche Forschungsgemeinschaft (DFG) in the framework of the German excellence initiative.

\section{References}

[1] S. Herold and D. Mayer: Actuators (2016) 5, 7.

[2] H. A. Sodano, G. Park and D. J. Inman: MSSP, 18, (2004) 683.

[3] J. Staeves: MP Mat. Test., 51, (2009) 48.

[4] M. Schwankl, S. Kimme, C. Pohle, W.-G. Drossel and C. Körner: Adv. Eng. Mater., 17, (2015) 969.

[5] S. Stein, J. Heberle, M. Suchy, F. Tenner, F. Hugger, S. Roth and M. Schmidt: J. Laser Micro/Nanoengin., 11, (2016) 111.

[6] B. Nogowizin: "Theorie und Praxis des Druckgusses" (Schiele \& Schön, Berlin, 2011).

[7] S. Amore, E. Ricci, T. Lanata and R. Novakovic: J. Alloys Compd., 452, (2008) 161.

[8] S. Stein, J. Wedler, S. Rhein, M. Schmidt, C. Körner, A. Michaelis, and S. Gebhardt: RINP, 7C, (2017) 2534.

[9] S. Stein, S. Roth, N. Modler, M. Gude, T. Weber and A. Winkler: Proceedings of 5th Scientific Symposium "Lightweight Design by Integrating Functions" Sep. 14-16 (2015) Dresden, Germany.
[10]K. Holeczek, E. Starke, A. Winkler, M. Dannemann, and N. Modler: Appl. Sci., 6, (2016) 55.

[11]W. A. Hufenbach, N. Modler, A. Winkler, J. Ilg and S. J. Rupitsch: Smart Mater. Struct., 23, (2014) 25011.

[12] A. Winkler and N. Modler N.: Mater. Sci. Forum, 825826, (2015) 787.

[13]P. Ribes, T. Burkhardt, M. Hornaff, S. Kousar, D. Burkhardt, E. Beckert, M. Gilaberte, D. Guilhot, M. Montes, S. Galan, R. Ferrando, A. Eberhardt and A. Tünnermann: Laser Ignition Conference (LIC'14), Yokohama, Japan, Apr. 22 - 24, 2014.

[14]A. Jeromen, C. Held, E. Govekar, S. Roth, and M. Schmidt: J. Mater. Process. Technol., 4, (2014) 737.

[15] R. C. Weast: "CRC Handbook of chemistry and physics” Ed by J. R. Rumble (CRC Press Ohio, 1974).

[16]W. Zhai, W. L. Wang, D. L. Geng and B. Wei: Acta Mater., 60, (2012) 6518.

[17]S. Stein, M. Dobler, T. Radel, M. Strauß, H. Breitschwerdt, F. Hugger, S. Roth and M. Schmidt: Production Engineering, 26, (2017) 1455.

[18] S. Stein, J. Heberle, F. J. Gürtler, K. Cvecek, S. Roth, and M. Schmidt.: Physics Procedia, 56, (2014) 709.

[19] J.-W. Kim and S.-B. Jung: Int. J. Solids. Struct., 43, (2006) 1928.

[20] J. F. Lancaster: "Metallurgy of Welding” (Woodhead, Cambridge, 2007).

[21] M. B. Uday, M. N. Ahmad-Fauzi, M. N. Alias, R. Srithar: "Current Issues and Problems in the Joining of Ceramic to Metal” (INTEC Publishing, Croatia, 2016).

[22]W. Kollenberg: "Technische Keramik. Grundlagen, Werkstoffe, Verfahrenstechnik”. (Vulkan, Essen, 2009).

[23]H.-T. Lee, M.-H. Chen, H.-M. Jao, and T.-L. Liao: Mater. Sci. Eng., A:, 358, (2003) 134.

(Received: August 7, 2017, Accepted: October 22, 2017) 